\title{
WBCs, Neutrophils to Lymphocytes, Monocytes to Lymphocytes and Platelets to Lymphocytes Ratios to Predict the Severity of Covid-19 Cases among Intensive Care Unit Patients
}

\section{Haytham Dahlwi*, Rana Zaini and Mazen Almehmadi}

Clinical Laboratory Sciences, Taif University, Saudi Arabia

*Corresponding Author: Haytham Dahlawi, Associate Professor, Hematology, Clinical Laboratory Department, College of Applied Medical Science, Taif University, Taif, Saudi Arabia.
Received: July 28, 2021

Published: August 13, 2021

(C) All rights are reserved by Haytham

Dahlwi., et al.

\begin{abstract}
Introduction: On March 2020, the World Health Organization (WHO) introduced the disease of Coronavirus 2019 (COVID-19) as a pandemic disorder. Although Covid-19 has spread rapidly causing serious health problems and might lead to death no effective treatment has discovered yet, the detection of reliable and useful prognostic markers is helpful to predict the severity and prognosis among Covid-19 patients. Thus, the current study aimed to assess and compare the level of WBCs, lymphocytes, neutrophils, monocytes and platelets in Covid-19 patients and control group. This study also evaluated neutrophils to lymphocytes, monocytes to lymphocytes and platelets to lymphocytes ratio.

Methods: This is a retrospective study included 385 participants 350 of which were confirmed COVID- 19 cases. Whereas the control group include 35 healthy individuals.

Results: The result of this study showed a significant increase in total WBCs count, significant low lymphocytes count and significant high neutrophil count in Covid-19 patients admitted in intensive care unit (ICU) compared to the control group. The result of this study also reported significantly $(\mathrm{P}<0.0001)$ higher values for neutrophil-to-lymphocyte ratio (NLR), monocytes-to-lymphocyte ratios (MLR) and platelet-to-lymphocyte ratio (PLR), in Covid-19 patients admitted in ICU.

Conclusion: Based on the findings of this study, which revealed significant changes in a number of blood cells among Covid-19 patients admitted to ICU, it is suggested that level of WBCs, NLR, MLR and PLR can be use as a useful prediction marker to predict the severity of Covid-19 cases in ICU.
\end{abstract}

Keywords: White Blood Cells; Neutrophils to Lymphocytes Ratio; Monocytes to Lymphocytes Ratio and Platelets to Lymphocytes Ratio; Covid-19

\section{Abbreviations}

NLR: Neutrophil-to-lymphocyte Ratio; MLR: Monocytes-to-lymphocyte Ratio; PLR: platelet-to-lymphocyte Ratio; ICU: Intensive Care Unit

\section{Introduction}

On March 2020, the World Health Organization (WHO) introduced the disease of Coronavirus 2019 (Covid-19) as a pandemic and a member of the Coronavirus family. Covid-19 found to be associated with different sign and symptoms ranging from the head- 
WBCs, Neutrophils to Lymphocytes, Monocytes to Lymphocytes and Platelets to Lymphocytes Ratios to Predict the Severity of Covid-19 Cases among Intensive Care Unit Patients

ache, fever, usual cold, fatigue, dry cough to respiratory failure, interstitial pneumonia and septic shock [1]. Such serious clinical manifestations are often develop rapidly, unexpected and might be fatal if not managed and treated urgently [1]. Individuals with chronic disease including hypertension, cancer, cardiovascular, pulmonary disorder and diabetes have been classified as high risk group [2,3]. Dysfunction of the immune cells as well as abnormal response were detected in patients with severe Covid-19, suggesting low concentration of neutrophil (NETs) within tissues [4,5]. Moreover, it has been reported that the low level of leukocytes or impaired their function could result in mild to severe health problem and disorders [6].

A number of several laboratory investigations have been assessed to predict the severity and prognosis among hospitalized Covid-19 patients. Such of theses hematological laboratory tests are C-reactive protein (CRP), white blood cell (WBC) level, red cell distribution width (RDW), ratio of neutrophil (NETs) to lymphocyte, ratio of monocytes to lymphocyte and ratio of platelet to lymphocyte [7]. A recent study done in Saudi Arabia has found a significant reduction in the concentration of total WBC, neutrophil, lymphocyte and monocyte count among patients with Covid-19 [1]. Lagunas-Rangel has evidenced that neutrophil-to-lymphocyte ratio (NLR), an important marker of systemic inflammation and infection, which improve the inflammatory function in Covid-19 patients [8]. A retrospective study included the clinical data from 443 Covid-19 patients with non-severe and sever cases at Wuhan Forth Hospital, found that neutrophil-to-lymphocyte ratio, CRP and platelets were independent protective factors for patients with severe Covid-19 [9]. Another retrospective analysis performed on 72 Covid-19 hospitalized patients reported low lymphocytes and low white blood cell count in $54.2 \%$ and $2027.8 \%$ patients respectively. Within the same study, it has been detected that leukocyte, neutrophil level and neutrophil-lymphocyte ratio (NLR) were significantly high in non-severe patients compared to patients with severe cases [10]. Udugama and colleagues found that age, WBC count and CRP level were significantly higher in severe Covid-19 patients compared to other patients, however, lymphocyte concentration was significantly lower [11]. The length of hospital stay and prognosis of the disease were noticed to be highly associated with increase in platelets-to-lymphocyte ratio PLR level among 30 hospitalized patients with confirmed Covid-19 in Huizhou Municipal Central Hospital [12].

\section{Aim of the Study}

The aim of this study was to assess the WBC indices, including total WBC level, neutrophil level, lymphocyte level, monocyte level and platelets count. This study was also evaluate the ratio of the following: neutrophils to lymphocytes, monocytes to lymphocytes and platelets to lymphocytes among Covid-19 cases at intensive care unit in Saudi Arabia.

\section{Materials and Methods}

This is a retrospective study included 350 confirmed Covid-19 adult patients who hospitalized in government hospitals at the Western region of Saudi Arabia. Out of these 350 patients, 197 (56\%) were mal and 153 (44\%) female. The majority were Saudi and aged above 40 year (Table 1). All patients were diagnosed by quantitative Polymerase Chain Reaction (qPCR) on collected nasopharyngeal swab. As the Saudi Ministry of Health criteria for intensive care unit admission, patients were symptomatic, a temperature of $38.5^{\circ} \mathrm{C}$ with a shortness of breath or decrease in mean arterial pressure below $60 \mathrm{mmHg}$. Most of ICU admitted patients were had a chronic disease including diabetics and hypertension.

\begin{tabular}{|l|c|c|c|}
\hline Characteristics & $\begin{array}{c}\text { Controls } \\
\text { (n= 35) }\end{array}$ & $\begin{array}{c}\text { CoVID-19 case } \\
\mathbf{n = 3 5 0 )} \\
\text { Median (95\%CI) } \\
\text { (95\%CI) }\end{array}$ & P-value \\
\hline Age & $64(59-66)$ & $64(64-64)$ & $0.1 \mathrm{NS}$ \\
\hline $\begin{array}{l}\text { White blood } \\
\text { Cells (WBC) }\end{array}$ & $8(7-9)$ & $9.9(9.2-10.4)$ & $0.001^{*}$ \\
\hline Lymphocyte & $3.3(3.2-4.0)$ & $1.3(1.2-1.4)$ & $<0: 0001^{* *}$ \\
\hline Platelet & $360(320-390)$ & $279(265-297)$ & $<0: 0001^{* *}$ \\
\hline Monocyte & $0.7(0.6-0.8)$ & $0.59(0.55-0.64)$ & $0.3 N S$ \\
\hline Neutrophil & $4.0(3.8-4.8)$ & $7.5(6.96-8.02)$ & $<0: 0001^{* *}$ \\
\hline NLR & $1.15(1.03-1.33)$ & $5.3(4.66-5.94)$ & $<0: 0001^{* *}$ \\
\hline MLR & $0.19(0.16-0.21)$ & $0.41(0.37-0.44)$ & $<0: 0001^{* *}$ \\
\hline PLR & 103.8 & $\begin{array}{c}207.9 \\
(190.3-224.8)\end{array}$ & $<0: 0001^{* *}$ \\
\hline
\end{tabular}

Table 1: Clinical characteristics and laboratory results of the control and covid-19 cohorts.

Patients demographic data were collected and results of hematological laboratory investigations were also recorded from 
the electronic medical records of the hospitals. Control group was including 35 healthy individuals 18 (51.4\%) females' participants and 17 (48.6\%) males) aged 40 year and older. Complete blood count (CBC) for the control group was recorded and the interested median ratios were calculated (Table 1). All personal identification information were kept highly confidential.

\section{Ethical approval}

The ethical approval for this study was obtained from the Research Ethics Committee at the College of Applied Medical science at Taif University as well as the Ethics Committee of the Ministry of Health (number:HAP-02-T-067). The study was conducted from March to September 2019.

\section{Statistical analysis}

Statistical analysis were carried under R statistical environment. Data distribution was tested using Shapiro-Wilk test of normality package. Non parametric version of t-tests to examine significance difference in median values was done using Wilcox test.

\section{Results}

The data set consists of 385 participants 350 of which were confirmed Covid- 19 cases admitted in ICU. There 153 females' patients (43.7\%) and 197 male patients (56.3\%) (Figure 1). Whereas the control group include 18 (51.4\%) females' participants and 17 (48.6\%) males.

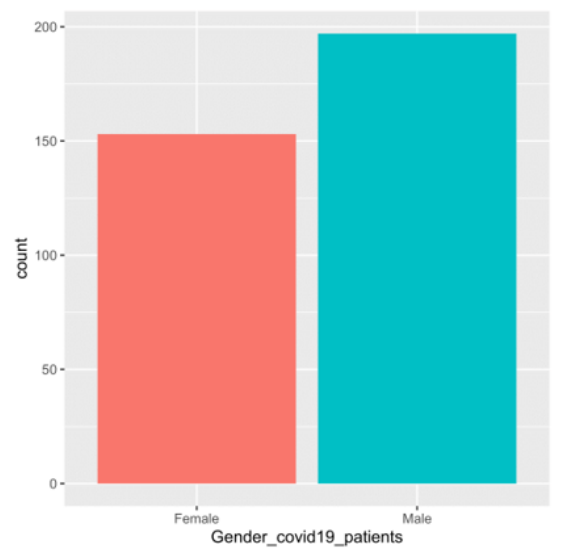

Figure 1: Distribution of Covid-19 patients' gender.
Table 1 shows clinical characteristics and laboratory results of the control group and Covid-19 cases as well as the median values with 95\% confidence interval and its significance differences. Equal medians of both patients' and control group age therefore no significance difference was attained (Figure 2).

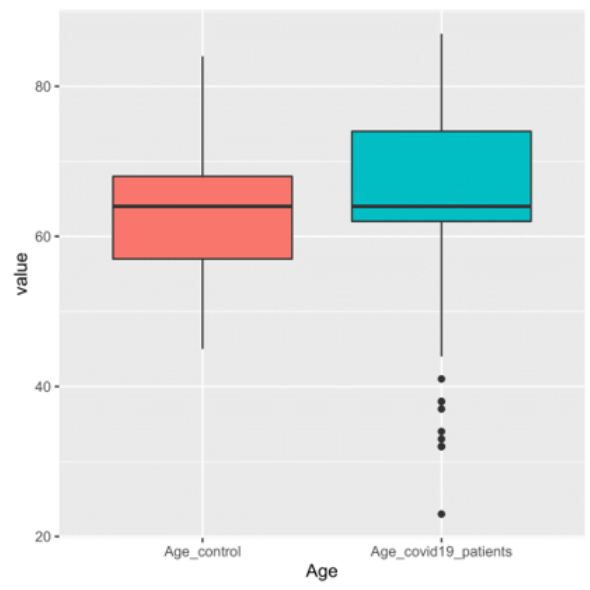

Figure 2: Age of control and Covid-19 patients.

Boxplot of Age for control and Covid-19 patients, where top and bottom lines represent the maximum and minimum values, the top and bottom of each box represent the first quartile (Q1) and third quartile (Q3) where the line inside each box is the median (second quartile Q2), dots blew are outlier value.

The Covid-19 patients in ICU had median of white blood cells (WBCs) count 9.9 which was significantly higher compared to the control group ( $\mathrm{p}-0.001)$. Figure 3A shows the Boxplot of white blood cells count of control and COVID-19 patients, where top and bottom lines represent the maximum and minimum values, the top and bottom of each box represent the first quartile (Ql) and third quartile $(\mathrm{Q} 3)$ where the line inside each box is the median (second quartile Q2), dots above are outlier values. The patients' group were showing great number of outlier values compared to the control group.

The result of this study showed that the Covid-19 patients in ICU had a median lymphocyte of 1.3 (Table 1) which was significantly lower compared to the control group $(\mathrm{P}<0.0001)$. It is also 


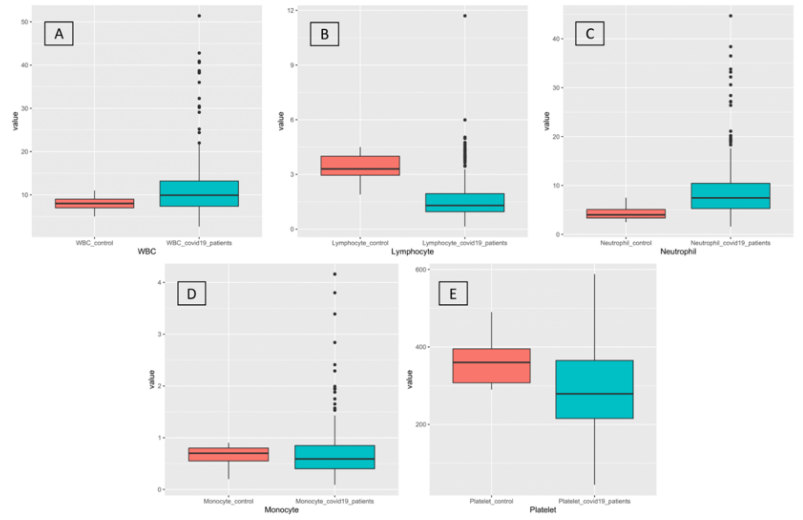

Figure 3: WBC, neutrophil, lymphocyte, monocyte and platelets of control and Covid-19 patients.

Boxplot of WBC, neutrophil, lymphocyte, monocyte and platelets for control and Covid-19 patients, where top and bottom lines represent the maximum and minimum values, the top and bottom of each box represent the first quartile (Q1) and third quartile (Q3) where the line inside each box is the median

(second quartile Q2), dots above are outlier value. worth mentioning the large number of outliers of Covid-19 patients (Figure 4B). A similar image was also observed for plateletto-lymphocyte ratio (PLR), where the Covid-19 patients had a median PLR of 207.9 (Table 1 and figure 4C) which was significantly $(\mathrm{P}<0.0001)$ higher compared to the control group (103.8). It is also worth mentioning the large number of outliers among Covid-19 patients.

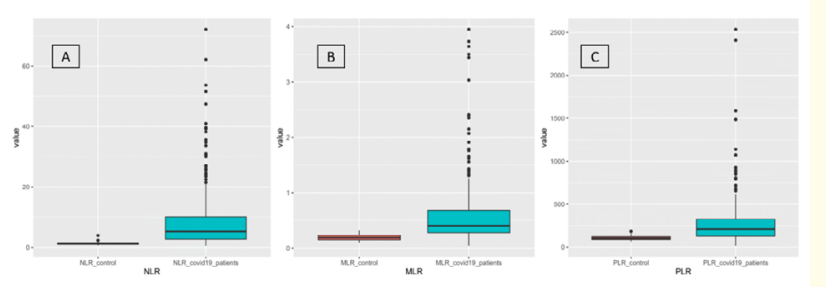

Figure 4: NLR, MLR, PLR of control and Covid-19 patients.

Boxplot of NLR, MLR, PLR of control and Covid-19 patients for control and Covid-19 patients, where top and bottom lines represent the maximum and minimum values, the top and bottom of each box represent the first quartile (Q1) and third quartile (Q3) where the line inside each box is the median (second quartile Q2), dots above are outlier value.

worth mentioning the large number of outliers among Covid-19 patients (Figure 3B). On the other hand, the median of neutrophil counts was significantly lower in control in comparison with Covid-19 patients 4.0 vs 7.5 (Table 1 and figure 3C). The current study also assessed the monocytes level among both groups, no significant differences were observed (Table 1 and figure 3D). In addition, statistically significant differences were observed in the platelet count ( $\mathrm{p}<0.0001$ ). Covid-19 patients admitted in ICU had a median of 279 compared to 360 for the controls (Table 1). No outliers' values were recorded of platelet counts for both controls and cases (Figure 3E).

Comparisons of neutrophil-to-lymphocyte ratios (NLR) between the different groups, showed values for control compared to Covid-19 patients significantly ( $\mathrm{P}<0.0001)$ lower $(1.15$ vs 5.3 ) (Figure 4A). The Covid-19 patients had a median monocytes-tolymphocyte ratios (MLR) of 5.3 (Table 1 ) which was significantly $(\mathrm{P}<0.0001)$ higher compared to the control group $(0.19)$. It is also

\section{Discussion}

Although Covid-19 has spread rapidly since 2019 and no effective treatment has discovered yet, the detection of reliable and useful prognostic markers is helpful to predict the severity and prognosis among Covid-19 patients [13]. Thus, the current study aimed to assess and compare the level of WBCs, lymphocytes, neutrophils, monocytes and platelets in Covid-19 patients and control group. This study also evaluated neutrophils to lymphocytes, monocytes to lymphocytes and platelets to lymphocytes ratio.

A significant increase in WBCs in Covid-19 patients admitted in ICU was reported in the present study. Unlike this study, $\mathrm{Xu}$ (2021) found out a lower WBC concentration in patients with Covid-19 compared to healthy individuals [14]. On the other hand, significantly higher WBC count was reported among 51 Covid-19 patients in ICU [15]. 
WBCs, Neutrophils to Lymphocytes, Monocytes to Lymphocytes and Platelets to Lymphocytes Ratios to Predict the Severity of Covid-19 Cases among Intensive Care Unit Patients

A research study suggesting that the inhibition on cellular immune function has been associated with the reduction in the level of lymphocytes, which coronavirus may cause [16]. The results of this study showed a significant low level of lymphocytes count and a significant high level of neutrophil counts in Covid-19 patients who admitted in ICU compared to the control group. Similar to this study, Eslamijouybari., et al. reported that lymphocyte counts were significantly lower and neutrophil counts were significantly higher in the Covid-19 positive cases compared to healthy persons [17]. Another more recent study found that lymphocyte and neutrophil counts were significantly low among Covid-19 patients, on admission [18].

Regarding monocytes count, some research study suggesting that monitoring this type of cell can be a simple and important prediction tool to identify the severity and risk of progression of Covid-19 [19]. The result of monocytes level in this study showed no significant differences among both groups. However, a study done in Italy detected low lymphocytes and monocytes count in the peripheral blood examination for Covid-19 patients [20]. Another study reported significantly less monocytes level and abnormal vacuolization under the microscopic examination for Covid-19 patients, who admitted in ICU [15].

The positive correlation has been found between the platelet level with the length of stay-in hospital among Covid-19 patients [21]. Jayanthi and Tulasi, in their prospective observational study showed that the decreased in platelet count associated with increased complication rate as well as study duration of hospital [22]. In this study, statistically significant decrease were observed in the platelet count $(\mathrm{p}<0.0001)$ in Covid-19 patients admitted in ICU. Similar to this study's result, a study reported a significant decrease in platelet count in patients with severe Covid-19 [23].

Focusing on neutrophil-to-lymphocyte ratio (NLR), monocytesto-lymphocyte ratios (MLR) and platelet-to-lymphocyte ratio (PLR), in this study, all three ratios were found to be significantly $(\mathrm{P}<0.0001)$ higher in Covid-19 patients admitted in ICU. In previous, a cross-sectional study has been assessed 527 patients with Covid-19 and found that neutrophil-to-lymphocyte ratio (NLR) was two times higher in the Covid-19 patients compared to controls, which is in line with this study [17]. The data were in line with this study's results reported in several research studies in different countries: Turkey [24], Saudi Arabia [1] and China [25].

\section{Conclusion}

The results of this study showed significant changes within the level of different parameters. Such of these are WBCs counts, lymphocytes, neutrophils and platelets level. In addition, statistically significant increase were detected on NLR, MLR and PLR. Thus, this study suggested that assessment of WBCs, NLR, MLR and PLR can be a useful prediction marker to predict the severity of Covid-19 cases who admitted to ICU.

\section{Limitations of the Study}

This study has number of limitations including the sample size was small and, hence, further, larger studies are warranted. Moreover, the study performed among limited hospital in the Westren region of Saudi Arabia, more area should be included.

\section{Declarations}

The authors declare that they have no conflict of interests.

\section{Bibliography}

1. Sayed A A A., et al. "The use of neutrophil-to-lymphocyte ratio (NLR) as a marker for COVID-19 infection in Saudi Arabia: A case-control retrospective multicenter study". (2021).

2. Guo Yan-Rong., et al. "The origin, transmission and clinical therapies on coronavirus disease 2019 (COVID-19) outbreakan update on the status". Military Medical Research 7.1 (2020): 1-10.

3. Rothan Hussin A and Siddappa N Byrareddy. "The epidemiology and pathogenesis of coronavirus disease (COVID-19) outbreak". Journal of Autoimmunity 109 (2020): 102433.]

4. R Ruan, Qiurong., et al. "Clinical predictors of mortality due to COVID-19 based on an analysis of data of 150 patients from Wuhan, China". Intensive Care Medicine 46.5 (2020): 846-848.

5. Cheng Olivia Z and Nades Palaniyar. "NET balancing: a problem in inflammatory lung diseases". Frontiers in Immunology 4 (2013): 1 .

6. Lamichhane Prem P and Amali E Samarasinghe. "The role of innate leukocytes during influenza virus infection". Journal of Immunology Research 2019 (2019).

7. Dahlawi Haytham. "Changes in Haematological Parameters among COVID-19 Patients". International Journal of Current 
WBCs, Neutrophils to Lymphocytes, Monocytes to Lymphocytes and Platelets to Lymphocytes Ratios to Predict the Severity of Covid-19 Cases among Intensive Care Unit Patients

Research and Review 12.18 (2020): 2-4.

8. LLagunas-Rangel Francisco Alejandro. "Neutrophil-to-lymphocyte ratio and lymphocyte-to-C-reactive protein ratio in patients with severe coronavirus disease 2019 (COVID-19): a meta-analysis". Journal of Medical Virology (2020).

9. Shang Weifeng., et al. "The value of clinical parameters in predicting the severity of COVID-19". Journal of Medical Virology 92.10 (2020): 2188-2192.

10. Ding Xiurong., et al. "Dynamic profile and clinical implications of hematological parameters in hospitalized patients with coronavirus disease 2019". Clinical Chemistry and Laboratory Medicine (CCLM) 58.8 (2020): 1365-1371.

11. Udugama Buddhisha., et al. "Diagnosing COVID-19: the disease and tools for detection". ACS Nano 14.4 (2020): 3822-3835.

12. Terpos Evangelos., et al. "Hematological findings and complications of COVID-19". American Journal of Hematology 95.7 (2020): 834-847.

13. Shivakumar BG., et al. "Neutrophil-to-Lymphocyte, Lymphocyte-to-Monocyte, and Platelet-to-Lymphocyte Ratios: Prognostic Significance in COVID-19”. Cureus 13.1 (2021).

14. Xu Xiaoping., et al. "Analysis of blood index characteristics in COVID-19 patients and their associations with different outcomes". Journal of Laboratory Medicine 45.3 (2021): 149-157.

15. Pozdnyakova Olga., et al. "Clinical significance of CBC and WBC morphology in the diagnosis and clinical course of COVID-19 infection". American Journal of Clinical Pathology 155.3 (2021): 364-375.

16. "Chen Nanshan., et al. "Epidemiological and clinical characteristics of 99 cases of 2019 novel coronavirus pneumonia in Wuhan, China: a descriptive study". The Lancet 395.10223 (2020): 507-513.

17. Eslamijouybari Mohammad., et al. "Neutrophil-to-Lymphocyte and Platelet-to-Lymphocyte Ratios in COVID-19 Patients and Control Group and Relationship with Disease Prognosis". Caspian Journal of Internal Medicine 11.1 (2020): 531.

18. Kamel Fatemah 0., et al. "Clinical and hematologic presentations of adults with COVID-19 patients in Jeddah: A case con- trol study". Journal of Infection and Public Health 14.6 (2021): 709-716.

19. Silvin Aymeric., et al. "Elevated calprotectin and abnormal myeloid cell subsets discriminate severe from mild COVID-19". Cell 182.6 (2020): 1401-1418.

20. Biamonte Flavia., et al. "Combined lymphocyte/monocyte count, D-dimer and iron status predict COVID-19 course and outcome in a long-term care facility". Journal of Translational Medicine 19.1 (2021): 1-10.

21. Qu Rong., et al. "Platelet-to-lymphocyte ratio is associated with prognosis in patients with coronavirus disease-19". Journal of Medical Virology 92.9 (2020): 1533-1541.

22. Jayanthi Hari Kishan and Sai Krishna Tulasi. "Correlation study between platelet count, leukocyte count, nonhemorrhagic complications, and duration of hospital stay in dengue fever with thrombocytopenia". Journal of Family Medicine and Primary Care 5.1 (2016): 120.

23. LLippi, Giuseppe, Mario Plebani, and Brandon Michael Henry. "Thrombocytopenia is associated with severe coronavirus disease 2019 (COVID-19) infections: a meta-analysis". Clinica Chimica Acta 506 (2020): 145-148.

24. Seyit Murat., et al. "Neutrophil to lymphocyte ratio, lymphocyte to monocyte ratio and platelet to lymphocyte ratio to predict the severity of COVID-19". The American Journal of Emergency Medicine 40 (2021): 110-114.

25. Kong Man., et al. "Higher level of neutrophil-to-lymphocyte is associated with severe COVID-19". Epidemiology and Infection $148(2020)$.

\section{Volume 5 Issue 9 September 2021 (C) All rights are reserved by Haytham Dahlwi., et al.}

